\title{
Quantum statistics of four-wave mixing by a nonlinear resonant microcavity
}

\author{
Y. Sherkunov, ${ }^{1}$ David M. Whittaker, ${ }^{2}$ Henning Schomerus, ${ }^{1}$ and Vladimir Fal'ko ${ }^{1}$ \\ ${ }^{1}$ Physics Department, Lancaster University, Lancaster, LA1 4YB England, United Kingdom \\ ${ }^{2}$ Department of Physics and Astronomy, University of Sheffield, Sheffield, S3 7RH England, United Kingdom
}

(Received 3 June 2014; published 29 September 2014)

\begin{abstract}
We analyze the correlation and spectral properties of two-photon states resonantly transmitted by a nonlinear optical microcavity. We trace the correlation properties of transmitted two-photon states to the decay spectrum of multiphoton resonances in the nonlinear microcavity.
\end{abstract}

DOI: 10.1103/PhysRevA.90.033845

\section{INTRODUCTION}

Generation and controlled propagation of two-photon states in optical circuits represent a new challenge in quantum optics-the next step from the earlier-developed single-photon sources. Photon pairs have been successfully produced using semiconductor quantum dots [1-5] and by coupling superconducting qubits to high-quality microwave resonators [6-12]. However, the controlled propagation of correlated photon pairs across optical circuits remains largely unexplored.

Here, we propose a theory describing the transmission of two-photon states in optical circuits where junctions are nonlinear optical or microwave resonators [13-16]. In such systems, the interaction between photons inside the cavity both shifts the conditions for their resonant transmission and leads to four-wave-mixing - a redistribution of photon energies in the transmitted pair. We show that the four-wave-mixing by the nonlinear cavity results in spectral correlations of the transmitted two-photon states, prescribed by the decay of multiphoton resonances in a nonlinear cavity, and we analyze quantum statistics of few-photon states emitted by a nonlinear resonator under the action of a weak classical pump.

\section{TRANSMISSION OF TWO-PHOTON STATES}

The key element of the optical circuits discussed in this paper is sketched in Fig. 1. It consists of a nonlinear microcavity, with resonance frequency $\omega_{c}$ and decay broadening $\gamma=\left(\gamma_{L}+\gamma_{R}\right) / 2=\omega_{c} / Q(Q$ stands for the quality factor $)$ determined by couplings $\gamma_{L(R)}$ to the left (right) waveguides. The interaction of two incoming photons of frequency $\omega_{0}$ inside the cavity generates two outgoing photons with frequencies $\omega$ and $\omega^{\prime}=2 \omega_{0}-\omega\left(\omega, \omega^{\prime} \approx \omega_{0}\right)$. As a function of the frequency of an outgoing photon $\omega$, the characteristic spectral density of the transmitted photons is illustrated by the color-scale plot in Fig. 1. The spectral density shows distinctive maxima at $\omega_{c}$ and $2 \omega_{0}-\omega_{c}$. These statements also apply to a nearly monochromatic pair in a two-photon pulse with a temporal extent of $\tau \gg \gamma^{-1}$. Figure 1 also shows that the four-wave mixing is most efficient either when the incoming photons' frequency resonates with the empty cavity mode or when the total energy $2 \hbar \omega_{0}$ of the incoming pair coincides with the energy of the interacting photon pair inside the cavity $2 \hbar\left(\omega_{c}+u\right)$, where $2 \hbar u$ is the interaction energy. Here, we show that spectral properties of the transmitted photon pair are similar over a broad range of cavity and incoming pulse parameters. The spectral density in Fig. 1 illustrates spectral properties of the resonant four-wave mixing, where, as we show below, the two-photon spectral function can be factorized
PACS number(s): 42.50.Ar, 42.50.Pq, 42.65.Yj

into an $\omega_{0}$-dependent probability to create a two-photon state in the cavity and the spectral density of the following decay of such a state due to the photon escape into the waveguides.

The evolution of photons transmitted by the nonlinear resonator sketched in Fig. 1 is modeled using the Hamiltonian:

$$
\begin{aligned}
H= & H_{0}+H_{u}+H_{L}+H_{R}, \\
H_{0}= & \omega_{c} \alpha^{\dagger} \alpha, \\
H_{u}= & u \alpha^{\dagger} \alpha^{\dagger} \alpha \alpha, \\
H_{L}= & \int d x\left\{\beta_{L}^{\dagger}(x)\left(-i \frac{\partial}{\partial x}\right) \beta_{L}(x)\right. \\
& \left.+\sqrt{\gamma_{L}} \delta(x)\left[\beta_{L}^{\dagger}(x) \alpha+\alpha^{\dagger} \beta_{L}(x)\right]\right\}, \\
H_{R}= & \int d x\left\{\beta_{R}^{\dagger}(x)\left(-i \frac{\partial}{\partial x}\right) \beta_{R}(x)\right. \\
& \left.+\sqrt{\gamma_{R}} \delta(x)\left[\beta_{R}^{\dagger}(x) \alpha+\alpha^{\dagger} \beta_{R}(x)\right]\right\},
\end{aligned}
$$

where $\alpha\left(\alpha^{\dagger}\right)$ is the annihilation (creation) operator of the resonant cavity mode with energy $\omega_{c}$ (here, fundamental constants are set to $c=1$ and $\hbar=1)$. The nonlinear term, $H_{u}$, in Eq. (1) with coupling constant $u$, leads to photonphoton interaction, shifting the two-photon state energy by $2 u$. This nonlinearity is generic for inversion-symmetric media; in particular, it can be the result of the repulsion between exciton polaritons in a microcavity with a large Rabi frequency [14-16]. The resonant cavity mode is coupled to the photons in the left $(L)$ and right $(R)$ semi-infinite waveguides, described by annihilation operators in the real-space representation, $\beta_{L(R)}(x)=\frac{1}{\sqrt{s}} \sum_{k>0} e^{i k x} \beta_{L(R)}(k)$ ( $k$ is the wave number and $s$ is the length of the waveguide), and $\beta_{L(R)}(x)$ are defined to describe incoming photons for $x<0$ and outgoing photons for $x>0$ (irrespective of the side of the resonator). The transformation of propagating waves into the cavity mode (and their reflection from the cavity) takes place at $x=0$.

The solution for the two-photon transmission or reflection problem is obtained by studying the evolution of the twophoton states $|\psi(t)\rangle$, governed by the Schrödinger equation, $i \partial_{t}|\psi(t)\rangle=H|\psi(t)\rangle$. We match the initial state to the incident photon pair in the left waveguide:

$$
|\psi\rangle_{t \rightarrow-\infty}=\frac{e^{i \omega_{0}\left(x_{1}+x_{2}-2 t\right)}}{\tau \sqrt{2}} f\left(x_{1}, x_{2}, t\right) \beta_{L}^{\dagger}\left(x_{1}\right) \beta_{L}^{\dagger}\left(x_{2}\right)|0\rangle,
$$

where the two-photon pulse envelope $f\left(x_{1}, x_{2}, t\right)$ has a temporal extent $\tau$ and is modeled using the scenarios listed in Table I. The projection of the transmitted or reflected photon pairs onto 

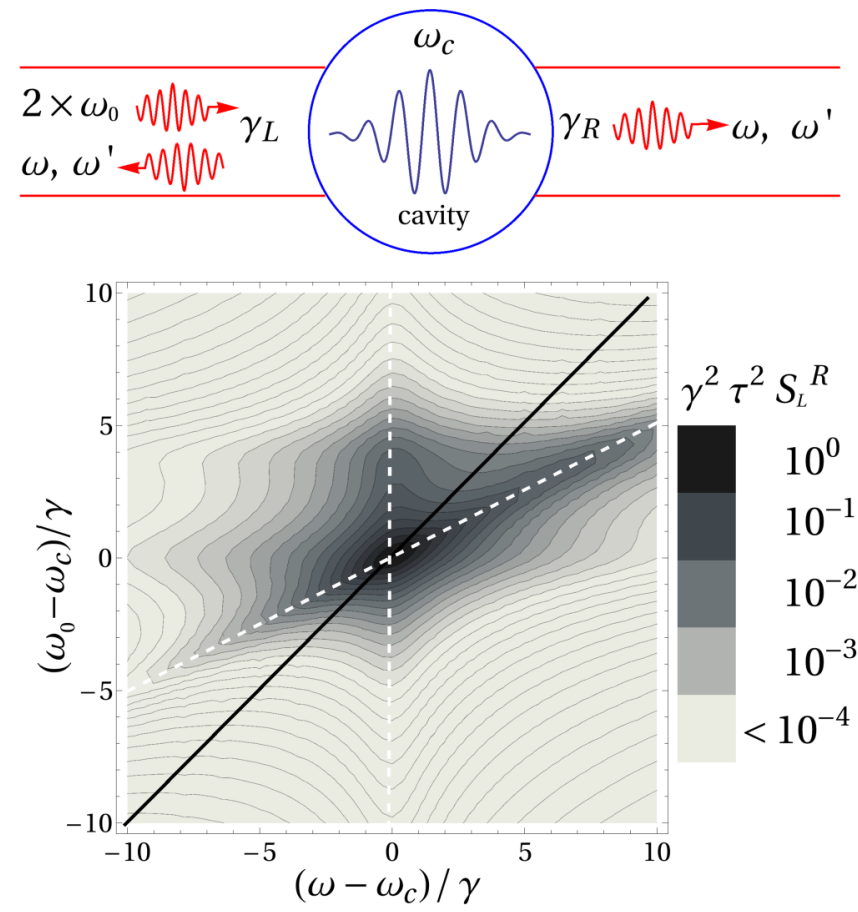

FIG. 1. (Color online) Two-photon transmission across a nonlinear resonator junction in an optical circuit. Top: Schematic of an optical cavity resonator connecting two waveguides. Bottom: Contour plot of spectral function $S_{L}^{R}$ characterizing spectral properties of transmitted two-photon states, as a function of frequencies $\omega$ of the outgoing photons and $\omega_{0}$ of the incident monochromatic photons, for $\gamma_{L}=\gamma_{R}=\gamma$ and nonlinear coupling $u / \gamma=4$. The single-photon transmission peak is indicated by the black line at $\omega=\omega_{0}$; maxima of $S_{L}^{R}$ at $\omega=\omega_{c}$ and $2 \omega_{0}-\omega_{c}$ are traced using the white dashed lines.

the outgoing states in the left or right leads $(i=L$ or $R$ ) is described using

$$
|\psi\rangle_{t \rightarrow \infty}=\frac{e^{i \omega_{0}\left(x_{1}+x_{2}-2 t\right)}}{\tau \sqrt{2}} a_{i j}\left(x_{1}, x_{2}, t\right) \beta_{i}^{\dagger}\left(x_{1}\right) \beta_{j}^{\dagger}\left(x_{2}\right)|0\rangle,
$$

where, for a monochromatic photon pair $(\tau \rightarrow \infty)$, the amplitudes $a_{L L}\left(a_{R R}\right)$ of obtaining two photons in the left (right) waveguide and $a_{L R}$ of finding one photon in each waveguide take the form (see Appendix A)

$$
\begin{aligned}
a_{i j} & =c_{i} c_{j}-\frac{u \gamma_{L} \sqrt{\gamma_{i} \gamma_{j}} e^{\left[i\left(\omega_{0}-\omega_{c}\right)-\gamma\right]\left|x_{1}-x_{2}\right|}}{\left(\omega_{0}-\omega_{c}+i \gamma\right)^{2}\left(\omega_{0}-\omega_{c}-u+i \gamma\right)}, \\
c_{L} & =-\frac{\omega_{0}-\omega_{c}+i\left(\gamma_{R}-\gamma_{L}\right) / 2}{\omega_{0}-\omega_{c}+i \gamma}, \\
c_{R} & =\frac{i \sqrt{\gamma_{L} \gamma_{R}}}{\omega_{0}-\omega_{c}+i \gamma} .
\end{aligned}
$$

Here, $c_{i}$ is a single-photon reflection $(L)$ and transmission $(R)$ amplitude in the circuit with a resonant cavity. The first term

TABLE I. Incident states.

\begin{tabular}{lc}
\hline \hline State & $f\left(x_{1}, x_{2}, t\right)$ \\
\hline (i) Monochromatic & 1 \\
(ii) Uncorrelated Gaussian & $\pi^{-1 / 2} e^{-\left[\left(x_{1}-t\right)^{2}+\left(x_{2}-t\right)^{2}\right] / 2 \tau^{2}}$ \\
(iii) Correlated Gaussian & $\pi^{-1 / 4} e^{-\left(x_{1}-x_{2}\right)^{2} / 2 \tau^{2}}$ \\
\hline \hline
\end{tabular}

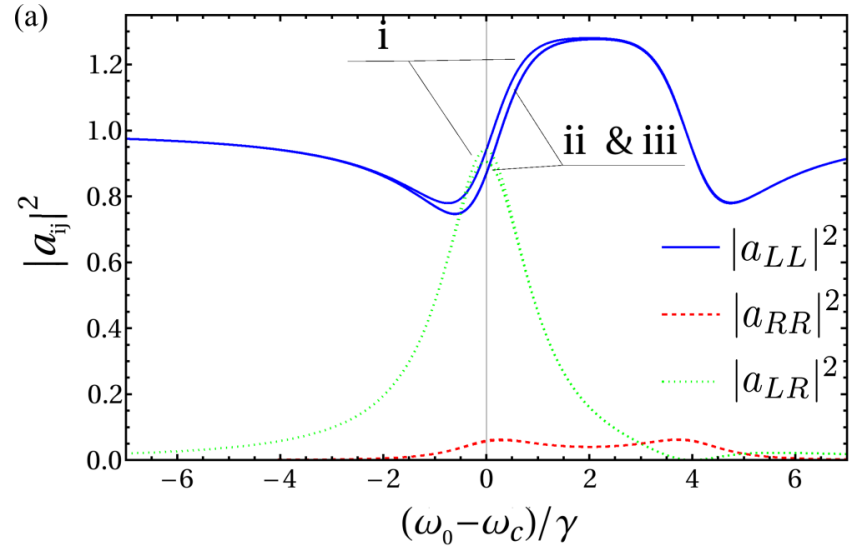

(b)

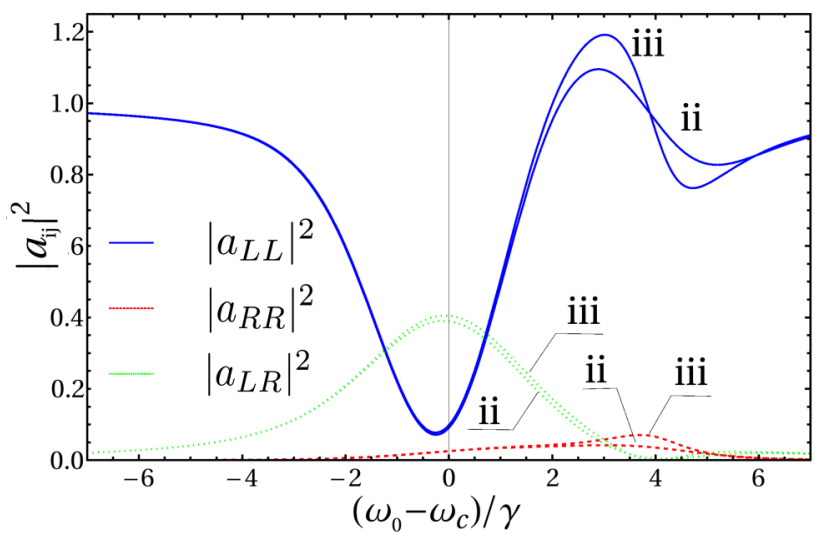

(c)

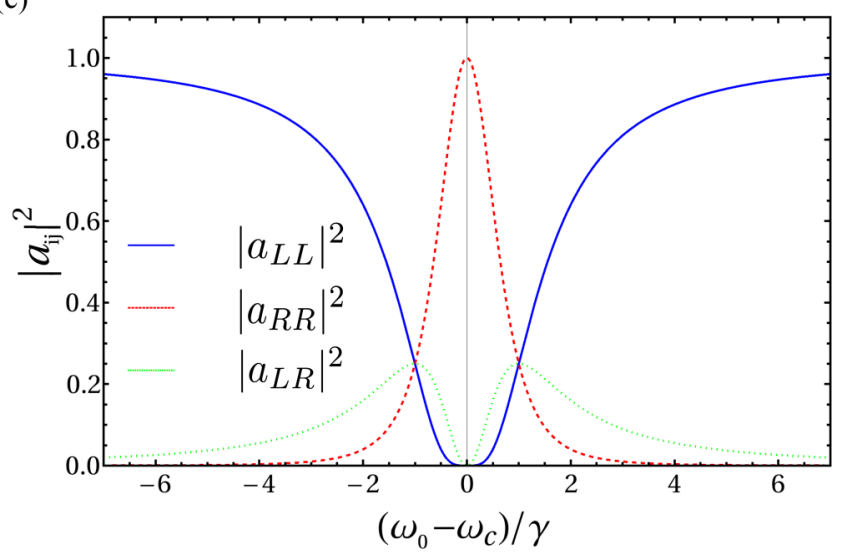

FIG. 2. (Color online) Reflection $\left|a_{L L}\right|^{2}$, transmission $\left|a_{R R}\right|^{2}$, and transmission-reflection $\left|a_{L R}\right|^{2}$ amplitudes of two-photon states in various parametric regimes. (a) Nonlinear cavity with $\tau \gamma=7$ $\left(\gamma_{L}=\gamma_{R}=\gamma\right)$ and $u=4 \gamma$. Frequency dependence of two-photon amplitudes $\left|a_{i j}\right|^{2}$ at $x_{1}=x_{2}$ for (i) a monochromatic incident wave, (ii) an uncorrelated initial pair of photons, and (iii) a correlated Gaussian initial state (see Table I). (b) Same as (a), but for $\tau \gamma=1$. (c) Transmission by a linear cavity $(u=0)$.

in Eq. (4) accounts for independent scattering of photons. The second term describes the correlated two-photon states formed due to the photon-photon interaction inside the cavity. Spectral properties of the amplitudes $a_{i j}$, calculated for various finite pulse widths, are illustrated in Fig. 2, where we show that the two-photon transmission by the nonlinear cavity is strongly affected by the photon-photon interaction, but does not depend 
much on the detailed spectral shape of the two-photon pulse. The examples in Figs. 2(a) and 2(b) demonstrate that the interaction of two photons inside the cavity produces their antibunching for $\omega_{0}=\omega_{c}$, but also generates an additional transmission peak at $\omega_{0}=\omega_{c}+u$ corresponding to the formation of resonant two-photon states inside the cavity. Note that in the limit $u \rightarrow \infty$ the model in Eq. (1) corresponds to the photon blockade regime studied in [17] for an optical junction consisting of a two-level system, and, in this limit, Eq. (4) reproduces the photon antibunching predicted in [17].

To characterize the spectral properties of the photon pair transmitted or reflected by the nonlinear cavity into a waveguide $i$ from a pulse arriving over a temporal interval $\tau$ (see Table I) in waveguide $j$, we consider the spectral density of photons detected at a distant position $x_{0} \rightarrow \infty$ in waveguide $i$. We assume a linear dispersion of the waveguide, which implies the following relation for the spatial and temporal variables $x=c t$, where $c$ is the speed of light:

$$
S_{j}^{i}(\omega) \equiv \int_{-\infty}^{\infty} \frac{d t}{2 \pi} e^{-i \omega t}\left\langle\beta_{i}^{\dagger}\left(x_{0}\right) \beta_{i}\left(x_{0}+t\right)\right\rangle_{j} .
$$

We find that the latter can be written in the form

$$
S_{j}^{i}(\omega)=\frac{\gamma_{j}^{2} \gamma_{i}}{\gamma^{3}} F\left(\omega_{0}\right) \tilde{F}(\omega)+I_{j}^{i} \delta\left(\omega-\omega_{0}\right),
$$

where the first term describes the photon-photon correlations introduced by the interaction between two photons simultaneously appearing inside the cavity, whereas the second term takes into account elastic (uncorrelated) single-photon transfer. In Eq. (5), the factor

$$
F\left(\omega_{0}\right)=\frac{2 \gamma^{2} / \pi \tau^{2}}{\left[\left(\omega_{0}-\omega_{c}\right)^{2}+\gamma^{2}\right]\left[\left(\omega_{0}-\omega_{c}-u\right)^{2}+\gamma^{2}\right]}
$$

is the probability to form the correlated two-photon state inside the cavity, whereas the factor

$$
\tilde{F}(\omega)=\frac{4 u^{2} \gamma^{2}}{\left[\left(\omega-\omega_{c}\right)^{2}+\gamma^{2}\right]\left[\left(\omega-2 \omega_{0}+\omega_{c}\right)^{2}+\gamma^{2}\right]}
$$

describes the spectral density of the photons emitted to the left or right waveguide. Finally, single-photon transfer is described by $I_{L}^{L}=I_{R}^{R}=2\left|c_{L}\right|^{2} / \tau$ and $I_{L}^{R}=I_{R}^{L}=2\left|c_{R}\right|^{2} / \tau$.

The weak dependence of the correlation properties of scattered photons on the correlation properties of the initial state seen in Fig. 2 indicates that the result in Eq. (5) is applicable to describe correlated photon pairs produced by incident two-photon states with different correlation properties, provided that $\tau \gamma \gg 1$.

\section{EMISSION OF FEW-PHOTON STATES UNDER THE ACTION OF A CLASSICAL PUMP}

The two-photon spectral correlations, described by Eq. (5), emerge even in the case of an initial state with different quantum statistics. To demonstrate this, we studied the emission spectrum by a nonlinear resonator excited by a coherent state $\mid$ in $\rangle=e^{-|b|^{2} / 2} e^{b \beta_{\omega_{0}}^{\dagger}}|0\rangle$ incident from one of the waveguides, e.g, $j=L$. Here, $|b|$ characterizes the amplitude $\langle$ in $|\beta|$ in $\rangle$ of the incoming field pulse. In this analysis, we exploit the possibility to relate the spectral function $S_{L}^{R}$ to the correlation function of the cavity resonance mode, as established in [18]:

$$
S_{L}^{R}(\omega)=\gamma_{R} \int_{-\infty}^{\infty} \frac{d t}{2 \pi} e^{-i \omega t} \operatorname{Tr} \alpha^{\dagger}(t) U(t, 0) \alpha(0) \rho(0),
$$

where the operators $\alpha$ and $\alpha^{\dagger}$ are used in the interaction representation, and $\rho$ is the reduced density matrix obtained from the full density matrix describing the system by tracing out the degrees of freedom associated with the quantum waveguide modes. The time dependence of the reduced density matrix $\rho$ obeys the Lindblad equation:

$$
\begin{aligned}
\partial_{t} \rho= & -i\left[H_{s}, \rho\right]+\gamma\left(2 \alpha \rho \alpha^{\dagger}-\alpha^{\dagger} \alpha \rho-\rho \alpha^{\dagger} \alpha\right), \\
\rho\left(t^{\prime}\right)= & U\left(t^{\prime}, t\right) \rho(t), \\
H_{s}= & \omega_{c} \alpha^{\dagger} \alpha+u \alpha^{\dagger} \alpha^{\dagger} \alpha \alpha \\
& +\left[\sqrt{\gamma_{L} / \tau} b e^{-i \omega_{0} t} \alpha^{\dagger}+\text { H.c. }\right],
\end{aligned}
$$

where $U\left(t^{\prime}, t\right)$ is the evolution superoperator of the reduced density matrix. When $U\left(t, t^{\prime}\right)$ is found using perturbation
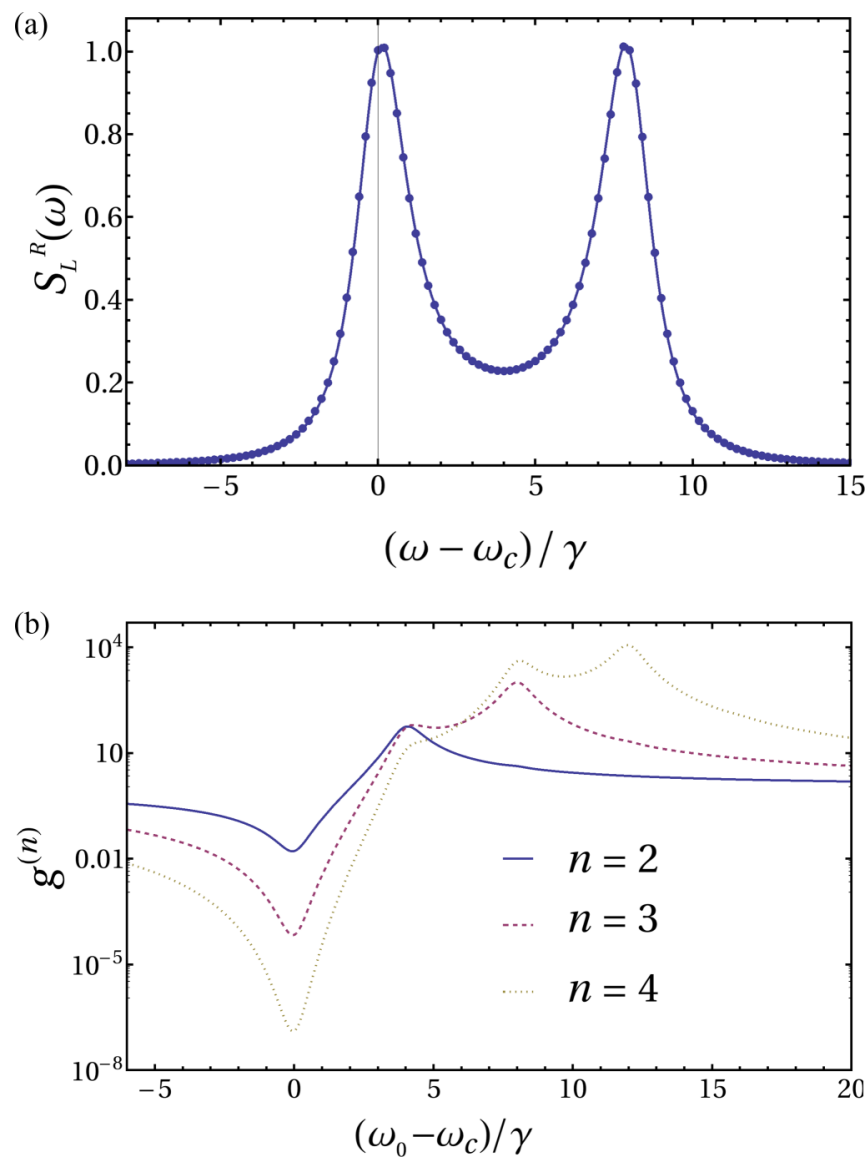

FIG. 3. (Color online) Generation of few-photon states in a nonlinear resonator excited by a classical field. (a) Spectral function $S_{L}^{R}(\omega)$ (normalized to its maximum value) for a cavity excited by a classical field source for $\gamma_{L}=\gamma_{R}=\gamma,\left(\omega_{0}-\omega_{c}\right) / \gamma=-4, u / \gamma=4$, $\sqrt{b / \gamma \tau}=0.1,\left(|\langle\alpha\rangle|^{2}=5.9 \times 10^{-4}\right)$. Dots represent the results of numerical calculations, and the solid line represents a fit to the spectral density described by Eq. (5). (b) Few-photon correlation function $g^{(n)}\left(\omega_{0}\right)$ for two-, three-, and four-photon states emitted to the right waveguide by a nonlinear optical resonator excited by a classical field arriving from the left waveguide. 
theory (see Appendix B) for a weak nonlinearity, $u \ll \gamma$, this results in the spectral density described by Eq. (5), but rescaled as $S_{L}^{R} \rightarrow|b|^{4} S_{L}^{R}$. A typical result of the numerical solution of Eq. (7) for a strong nonlinearity but weak pumping $\left(\left\langle\alpha^{\dagger} \alpha\right\rangle \ll 1\right)$ is shown in Fig. 3. It indicates that in the case of weak coherent pumping the spectrum of emitted photons is determined by two-photon correlations since its density coincides with that described by Eq. (5).

In addition, we analyze the conditions for the generation of correlated few-photon states with two, three, and four photons for a nonlinear cavity excited by a classical field. The appearance of such states is quantified using the correlation function:

$$
g^{(n)}=\left\langle\left(\beta_{i}^{\dagger}\right)^{n} \beta_{i}^{n}\right\rangle /\left\langle\beta_{i}^{\dagger} \beta_{i}\right\rangle^{n},
$$

where $\beta$ operators are taken at coinciding times. By generalizing the analysis in [18], this can be expressed in terms of the photon operators inside the cavity:

$$
g^{(n)}=\operatorname{Tr}\left[\left(\alpha^{\dagger}\right)^{n}(\alpha)^{n} \rho\right] / \operatorname{Tr}\left[\alpha^{\dagger} \alpha\right]^{n},
$$

which can be determined from Eq. (7). The results of numerical simulations for $n=2,3,4$ are shown in Fig. 3(b). Here, the second-order correlation function shows antibunching $\left(g^{(2)}<1\right)$ for red-detuned pumping frequencies and bunching $\left(g^{(2)}>1\right)$ for blueshifted frequencies, in agreement with [15]. Also, the functions $g^{(n)}$ peak at pumping frequencies close to $\omega_{0}=\omega_{c}+(n-1) u$, which correspond to the resonance conditions for a simultaneous excitation of $n$ photons inside the nonlinear cavity.

\section{CONCLUSIONS}

To summarize, we show that two-photon states resonantly transmitted by a nonlinear optical microcavity display spectral correlations, which are almost independent of the correlation properties of the incoming photon pair and can be traced to the decay spectrum of multiphoton resonances in the cavity.

\section{ACKNOWLEDGMENT}

We thank B. Altshuler, J. Bloch, A. Cleland, Y. Pashkin, and M. Skolnick for useful discussions. This work was supported by the Engineering and Physical Sciences Research Council Program Grant No. EP/J007544.

\section{APPENDIX A: PROPAGATION OF TWO-PHOTON STATES: SCATTERING APPROACH}

A generic two-photon state $|\psi(t)\rangle$, whose evolution obeys $i \frac{\partial}{\partial t}|\psi(t)\rangle=H|\psi(t)\rangle$, can be written as

$$
\begin{aligned}
|\psi(t)\rangle= & 1 / \sqrt{2}\left[\int d x_{1} d x_{2} \psi_{L L}\left(x_{1}, x_{2}, t\right) \beta_{L}^{\dagger}\left(x_{1}\right) \beta_{L}^{\dagger}\left(x_{2}\right)|0\rangle+\int d x_{1} d x_{2} \psi_{R R}\left(x_{1}, x_{2}, t\right) \beta_{R}^{\dagger}\left(x_{1}\right) \beta_{R}^{\dagger}\left(x_{2}\right)|0\rangle\right] \\
& +\sqrt{2} \int d x_{1} d x_{2} \psi_{L R}\left(x_{1}, x_{2}, t\right) \beta_{L}^{\dagger}\left(x_{1}\right) \beta_{R}^{\dagger}\left(x_{2}\right)|0\rangle \\
& +\int d x\left[\psi_{L C}(x, t) \beta_{L}^{\dagger}(x) \alpha^{\dagger}+\psi_{R C}(x, t) \beta_{R}^{\dagger}(x) \alpha^{\dagger}\right]|0\rangle+1 / \sqrt{2} \psi_{C C}(t) \alpha^{\dagger} \alpha^{\dagger}|0\rangle .
\end{aligned}
$$

Here, $|0\rangle$ is the vacuum state and $\psi_{i j}$ are the wave functions describing photons in the left (right) waveguide $[i, j=L(R)]$ or in the cavity $(i, j=C)$. This leads to the following system of simultaneous equations:

$$
\begin{aligned}
i\left(\partial_{x_{1}}+\partial_{x_{2}}+\partial_{t}\right) \psi_{i j}\left(x_{1}, x_{2}, t\right) & =1 / \sqrt{2}\left[\kappa_{i} \delta\left(x_{1}\right) \psi_{j C}\left(x_{2}, t\right)+\kappa_{j} \delta\left(x_{2}\right) \psi_{i C}\left(x_{1}, t\right)\right], \\
i\left(\partial_{x}+\partial_{t}+i \omega_{c}\right) \psi_{i C}(x, t) & =\sqrt{2}\left\{\kappa_{i}\left[\delta(x) \psi_{C C}(t)+\psi_{i i}(0, x, t)\right]+\kappa_{j} \psi_{i j}(x, 0, t)\right\}, \quad i \neq j, \\
i\left[\partial_{t}+i 2\left(\omega_{c}+u\right)\right] \psi_{C C}(t) & =\sqrt{2}\left[\kappa_{L} \psi_{L C}(0, t)+\kappa_{R} \psi_{R C}(0, t)\right] .
\end{aligned}
$$

For the incident state $|\psi\rangle_{t \rightarrow-\infty}=\frac{1}{\tau \sqrt{2}} e^{i \omega_{0}\left(x_{1}+x_{2}-2 t\right)} f\left(x_{1}, x_{2}, t\right) \beta_{L}^{\dagger}\left(x_{1}\right) \beta_{L}^{\dagger}\left(x_{2}\right)|0\rangle$ [see Eq. (2)] and $\psi_{C C}(t \rightarrow-\infty)=\psi_{L C}(t \rightarrow$ $-\infty)=\psi_{R C}(t \rightarrow-\infty)=0$, we find that

$$
\begin{aligned}
& \psi_{L L}\left(x_{1}, x_{2}, t\right)=\frac{e^{i \omega_{0}\left(x_{1}+x_{2}-2 t\right)}}{\tau}\left\{f\left(x_{1}, x_{2}, t\right) \theta\left(-x_{1}\right) \theta\left(-x_{2}\right)+a_{L L}\left(x_{1}, x_{2}, t\right) \theta\left(x_{1}\right) \theta\left(x_{2}\right)+c_{L}\left[\theta\left(x_{1}\right) \theta\left(-x_{2}\right)+\theta\left(-x_{1}\right) \theta\left(x_{2}\right)\right]\right\}, \\
& \psi_{R R}\left(x_{1}, x_{2}, t\right)=\frac{e^{i \omega_{0}\left(x_{1}+x_{2}-2 t\right)}}{\tau} a_{R R}\left(x_{1}, x_{2}, t\right) \theta\left(x_{1}\right) \theta\left(x_{2}\right), \\
& \psi_{L R}\left(x_{1}, x_{2}, t\right)=\frac{e^{i \omega_{0}\left(x_{1}+x_{2}-2 t\right)}}{\tau}\left[a_{L R}\left(x_{1}, x_{2}, t\right) \theta\left(x_{1}\right) \theta\left(x_{2}\right)+c_{R} \theta\left(-x_{1}\right) \theta\left(x_{2}\right)\right],
\end{aligned}
$$

where $a_{L L}, a_{R R}$, and $a_{L R}$ are the amplitudes describing two reflected photons, two transmitted photons, and one reflected and one transmitted photon, respectively. In the case of a monochromatic incident wave $(\tau \gamma \gg 1)$ (i), they are given by

$$
\begin{aligned}
a_{i j}\left(x_{1}, x_{2}\right) & =c_{i} c_{j}-\frac{u \gamma_{L} \sqrt{\gamma_{i} \gamma_{j}} \exp \left\{\left[i\left(\omega_{0}-\omega_{c}\right)-\gamma\right]\left|x_{1}-x_{2}\right|\right\}}{\left(\omega_{0}-\omega_{c}+i \gamma\right)^{2}\left(\omega_{0}-\omega_{c}-u+i \gamma\right)}, \\
c_{L} & =-\frac{\omega_{0}-\omega_{c}+i\left(\gamma_{R}-\gamma_{L}\right) / 2}{\omega_{0}-\omega_{c}+i \gamma}, \\
c_{R} & =\frac{i \sqrt{\gamma_{L} \gamma_{R}}}{\omega_{0}-\omega_{c}+i \gamma} .
\end{aligned}
$$




\section{APPENDIX B: CLASSICAL PUMPING, WEAK INTERACTION LIMIT}

In the case of weak nonlinearity $\left\langle\alpha^{\dagger} \alpha\right\rangle u \ll \gamma$, Eq. (7) can be solved analytically by expanding the density matrix over a coherent basis $|a\rangle$ so that $\rho=\int P(a)|a\rangle\langle a| d(\operatorname{Re}\{a\}) d(\operatorname{Im}\{a\})$ ( $P$ representation) [18] and converting into a Fokker-Planck equation with the help of the operator equivalence relations: $\alpha \rho \rightarrow a P(a), \alpha^{\dagger} \rho \rightarrow\left(a^{*}-\partial_{a}\right) P(a), \rho \alpha \rightarrow\left(a-\partial_{a^{*}}\right) P(a)$, and $\rho \alpha^{\dagger} \rightarrow a^{*} P(a)$. Switching to the rotating frame $(a \rightarrow$ $\left.a e^{-i \omega_{0} t}\right)$, we find

$$
\frac{\partial P}{\partial t}=-\sum_{i} \frac{\partial}{\partial a_{i}} B_{i} P+\frac{1}{2} \sum_{i j} \frac{\partial^{2}}{\partial a_{i} \partial a_{j}} D_{i j} P,
$$

where $\quad \boldsymbol{a}=\left(\begin{array}{c}a \\ a^{*}\end{array}\right), \quad \boldsymbol{B}=\left(\begin{array}{c}i a\left(\omega_{0}-\omega_{c}-2 u|a|^{2}+i \gamma\right)-i f \\ -i a^{*}\left(\omega_{0}-\omega_{c}-2 u|a|^{2}-i \gamma\right)+i f\end{array}\right), \quad D=$ $\left(\begin{array}{cc}-2 i u a^{2} & 0 \\ 0 & 2 i u a^{* 2}\end{array}\right)$, and $f=\sqrt{\gamma_{L} / \tau b}$. Equation (B1) is equivalent to the Langevin equation [18]:

$$
\partial_{t} \boldsymbol{a}=\boldsymbol{B}+\boldsymbol{\zeta}(t)
$$

where $\zeta_{i}(t)$ are delta-correlated random noise terms so that $\left\langle\zeta_{i}(t) \zeta_{j}\left(t^{\prime}\right)\right\rangle=D_{i j} \delta\left(t-t^{\prime}\right)$.
For small fluctuations, we can expand $a$ around the ensemble average $\langle a\rangle$, which satisfies the steady-state equation $|\langle a\rangle|^{2}\left[\gamma^{2}+\left(\omega_{0}-\omega_{c}-2 u|\langle a\rangle|^{2}\right)^{2}\right]=|f|^{2}$. We introduce new real variables, $r$ and $\theta$, so that $a=\langle a\rangle(1+r-i \theta)$, and rewrite Eq. (B2) for $\mathbf{X}=\left(\begin{array}{c}r \\ \theta\end{array}\right)$ as

$$
\partial_{t} \mathbf{X}=A \mathbf{X}+\boldsymbol{\eta}(t)
$$

where $\quad A=\left(\begin{array}{cc}-\gamma & \omega_{0}-\omega_{c}-2|\langle a\rangle|^{2} u \\ -\left(\omega_{0}-\omega_{c}-6|\langle a\rangle|^{2} u\right) & -\gamma\end{array}\right), \quad \boldsymbol{\eta}=$ $\frac{1}{2}\left(\begin{array}{c}\zeta_{1} /\langle a\rangle+\zeta_{2} /\left\langle a^{*}\right\rangle \\ i\left(\zeta_{1} /\langle a\rangle-\zeta_{2} /\left\langle a^{*}\right\rangle\right)\end{array}\right)$. For the initial condition $X(0)=0$, Eq. (B3) has the solution

$$
\mathbf{X}(t)=e^{A t} \int_{0}^{t} e^{-A t^{\prime}} \boldsymbol{\eta}\left(t^{\prime}\right) d t^{\prime},
$$

which for the spectral function $S_{L}^{R}(\omega)$ [Eq. (6)] yields

$$
S_{L}^{R}(\omega)=|b|^{4}\left(I_{L}^{R} \delta\left(\omega-\omega_{0}\right)+\frac{\gamma_{L}^{2} \gamma_{R}}{\gamma^{3}} F\left(\omega_{0}\right) \tilde{F}(\omega)\right),
$$

where $F\left(\omega_{0}\right)$ and $\tilde{F}(\omega)$ are given below Eq. (5).
[1] R. M. Stevenson et al., Nature (London) 439, 179 (2006).

[2] N. Akopian, N. H. Lindner, E. Poem, Y. Berlatzky, J. Avron, D. Gershoni, B. D. Gerardot, and P. M. Petroff, Phys. Rev. Lett. 96, 130501 (2006).

[3] C. L. Salter et al., Nature (London) 465, 594 (2010).

[4] A. J. Bennett et al., Nature Phys. 6, 947 (2010).

[5] R. M. Stevenson, C. L. Salter, J. Nilsson, A. J. Bennett, M. B. Ward, I. Farrer, D. A. Ritchie, and A. J. Shields, Phys. Rev. Lett. 108, 040503 (2012).

[6] A. Wallraff et al., Nature (London) 431, 162 (2004).

[7] D. I. Schuster et al., Nature (London) 445, 515 (2007).

[8] M. Hofheinz et al., Nature (London) 454, 310 (2008).

[9] M. Hofheinz et al., Nature (London) 459, 546 (2009).

[10] M. Mariantoni et al., Nature Phys. 7, 287 (2011).
[11] M. H. Devoret and R. J. Schoelkopf, Science 339, 1169 (2013).

[12] Y. Yin et al., Phys. Rev. Lett. 110, 107001 (2013).

[13] K. Nozaki et al., Nature Photon. 6, 248 (2012).

[14] H. S. Nguyen, D. Vishnevsky, C. Sturm, D. Tanese, D. Solnyshkov, E. Galopin, A. Lemaître, I. Sagnes, A. Amo, G. Malpuech, and J. Bloch, Phys. Rev. Lett. 110, 236601 (2013).

[15] A. Verger, C. Ciuti, and I. J. Carusotto, Phys. Rev. B 73, 193306 (2006).

[16] I. Carusotto, D. Gerace, H. E. Tureci, S. De Liberato, C. Ciuti, and A. Imamoğlu, Phys. Rev. Lett. 103, 033601 (2009).

[17] J.-T. Shen and S. Fan, Phys. Rev. Lett. 98, 153003 (2007).

[18] D. F. Walls and G. J. Milburn, Quantum Optics (Springer-Verlag, Berlin, 1994). 